\title{
A topological metric in $2+1$ dimensions
}

\author{
S. Habib Mazharimousavi ${ }^{\mathrm{a}}$, M. Halilsoy ${ }^{\mathrm{b}}$ \\ Department of Physics, Eastern Mediterranean University, Gazimag̃usa, Turkey
}

Received: 15 April 2015 / Accepted: 25 May 2015 / Published online: 4 June 2015

(C) The Author(s) 2015. This article is published with open access at Springerlink.com

\begin{abstract}
A real-valued triplet of scalar fields as a source gives rise to a metric which tilts the scalar, not the light cone, in $2+1$ dimensions. The topological metric is static, regular, and it is characterized by an integer $\kappa= \pm 1, \pm 2, \ldots$ The problem is formulated as a harmonic map of Riemannian manifolds in which the integer $\kappa$ equals the degree of the map.
\end{abstract}

\section{Introduction}

The topic of metrical kinks has a long history in general relativity [1-8] which declined recently toward oblivion. On the other hand in a broader sense interest in topological aspects in non-linear field theory, for a number of reasons, remains ever alive. Although these emerge mostly in flat $3+1$-dimensional spacetime, with the advent of higher/lower dimensions the same topological concepts may find applications in these cases as well.

The aim of this paper is to revisit this subject in $2+1$ dimensions. Motivation for this lies in part by the discovery of a cosmological black hole $[9,10]$ which became a center of attraction in this particular dimension. Does a topological metric also make a black hole? The answer to this question turns out to be negative, at least in our present study. The derived metric is sourced by a triplet of scalar fields $\phi^{a}(r, \theta)(a=1,2,3$, is the internal index $)$ which satisfies the constraint $\left(\phi^{a}\right)^{2}=1$, with topological properties. Let us add that besides the topological solution our system of triplet fields admits special solutions, including black holes. Our interest, however, will be focused on the topological one. Unlike the geometrical kink metrics [1-8] which tilt the light cone, leading to closed timelike curves, our topological metric tilts the scalar field along its range. The metric admits an integer, $\kappa= \pm 1, \pm 2, \ldots$, which can be interpreted (fol-

\footnotetext{
a e-mail: habib.mazhari@emu.edu.tr

b e-mail: mustafa.halilsoy@emu.edu.tr
}

lowing Ref. [11]) as the topological charge/homotopy class. The total energy is a multiple of $|\kappa|$ and the relation with the harmonic maps of Riemannian manifolds [12] suggests that $\kappa$ is at the same time the degree of the map. Let us note that Ref. [11] was extended shortly afterwards in a detailed analysis by Clement [13]. In search for a topological particle interpretation, Clement gave models and exact solutions in lower dimensions that can further be generalized to higher dimensions. That is, in $n+1$ dimensions, an $n$-scalar field model can be considered in a flat spacetime without much difficulty. Similar considerations in a curved spacetime with gravitation naturally add their own complications. In particular, the solution given in [13] for $2+1$ dimensions with gravitation relates closely to our study, which happens yet to be different from what has been considered here.

\section{The formalism}

In the $2+1$-dimensional spacetime

$\mathrm{d} s^{2}=-A(r) \mathrm{d} t^{2}+\frac{\mathrm{d} r^{2}}{B(r)}+r^{2} \mathrm{~d} \theta^{2}$

we choose the action $(16 \pi G=c=1)$

$I=\int \mathrm{d}^{3} x \sqrt{-g}\left(R-\frac{1}{2}\left(\nabla \phi^{a}\right)^{2}-\frac{\lambda}{2}\left(\left(\phi^{a}\right)^{2}-1\right)\right)$

in which

$\phi^{a}(r, \theta)=\left(\begin{array}{c}\sin \alpha(r) \cos \beta(\theta) \\ \sin \alpha(r) \sin \beta(\theta) \\ \cos \alpha(r)\end{array}\right)$.

Our notation is as follows: $R$ is the Ricci scalar, $A(r), B(r)$, and $\alpha(r)$ are functions of $r, \beta(\theta)$ is a function of $\theta$ and $\lambda(r, \theta)$ is a Lagrange multiplier. $\phi^{a}(r, \theta)$ transforms under the symmetry group $O(3)$ and satisfies

$\phi^{a} \phi^{a}=1$. 
The variational principle yields the field equation

$\square \phi^{a}=\lambda \phi^{a}$

in which $\square$ stands for the covariant Laplacian, and we have the constraint condition (4).

In the sequel we shall make the choice

$\beta(\theta)=\kappa \theta$

with $\kappa= \pm$ (integer) chosen for the uniqueness condition. This reduces the action effectively, modulo the time sector, to

$I=\int r \mathrm{~d} r \sqrt{\frac{A}{B}}\left(R-\frac{1}{2} B \alpha^{\prime 2}-\frac{\kappa^{2}}{2 r^{2}} \sin ^{2} \alpha\right)$

in which a prime stands for $\frac{\mathrm{d}}{\mathrm{d} r}$. With the energy-momentum tensor

$T_{\mu}^{v}=\frac{1}{2}\left[\left(\partial_{\mu} \phi^{a}\right)\left(\partial^{v} \phi^{a}\right)-\frac{1}{2}\left(\nabla \phi^{a}\right)^{2} \delta_{\mu}^{v}\right]$,

a variation with respect to $\alpha(r)$, and using the Einstein equations

$G_{\mu}^{v}=T_{\mu}^{v}$

we obtain the following equations:

$\left(r \sqrt{A B} \alpha^{\prime}\right)^{\prime}=\frac{\kappa^{2}}{2 r} \sqrt{\frac{A}{B}} \sin 2 \alpha$,

$\frac{-2 B^{\prime}}{r}=B \alpha^{\prime 2}+\frac{\kappa^{2}}{r^{2}} \sin ^{2} \alpha$,

$\frac{2 B A^{\prime}}{r A}=B \alpha^{\prime 2}-\frac{\kappa}{r^{2}} \sin ^{2} \alpha$,

$2 A^{\prime \prime}-\frac{A^{\prime 2}}{A}+\frac{A^{\prime} B^{\prime}}{B}=-\frac{A}{B}\left(B \alpha^{\prime 2}-\frac{\kappa^{2}}{r^{2}} \sin ^{2} \alpha\right)$.

This system of differential equations admits a number of particular solutions. As examples we give the following.

\subsection{A black hole solution}

A black hole solution is obtained by

$\alpha=\frac{\pi}{2}$

$A(r)=B(r)=C_{0}-\frac{\kappa^{2}}{2} \ln r$

where $C_{0}$ is an integration constant that can be interpreted as a mass. The scalar field triplet takes the form

$\phi^{a}(\theta)=\left(\begin{array}{c}\cos \kappa \theta \\ \sin \kappa \theta \\ 0\end{array}\right)$, which is effectively a doublet of scalars. The Ricci scalar of this solution reads

$R=\frac{\kappa^{2}}{2 r^{2}}$,

which is singular at $r=0$. The event horizon $r_{h}$ of the resulting black hole is

$r_{h}=\mathrm{e}^{2 C_{0} / \kappa^{2}}$

so that it is characterized by the index $\kappa$. Similarly the Hawking temperature also is determined by the integer $\kappa^{2}$. Clearly this is a different situation from the Bañados-TeitelboimZanelli (BTZ) black hole [9,10], where the parameter, i.e. the cosmological constant is not an integer (and neither is the electric charge).

\subsection{The topological solution}

Our system of equations (10), (11), (12), and (13) admits a solution with the choice $A(r)=1$. Accordingly, (10) reduces to the sine-Gordon equation

$(2 \alpha)_{u и}=\sin (2 \alpha)$

where

$\mathrm{e}^{2 u}=\left(\frac{r_{0}}{r}\right)^{2}-1$

in which $r_{0}$ is a constant that will be set simply to $r_{0}=1$. In the new variable

$\rho=\tanh ^{-1} r$.

the solutions for $\alpha(\rho)$ and $B(\rho)$ become

$\alpha(\rho)=2 \tan ^{-1}\left(\frac{1}{\sinh \rho}\right)$,

$B(\rho)=\frac{\kappa^{2}}{\cosh ^{4} \rho}$,

so that the resulting $2+1$-dimensional line element takes the form

$\mathrm{d} s^{2}=-\mathrm{d} t^{2}+\frac{\mathrm{d} \rho^{2}}{\kappa^{2}}+\tanh ^{2} \rho \mathrm{d} \theta^{2}$,

which can be cast through the transformation

$\sinh \rho=\left(\frac{r}{r_{0}}\right)^{\kappa}$

into the form

$\mathrm{d} s^{2}=-\mathrm{d} t^{2}+\frac{\left(\mathrm{d} r^{2}+r^{2} \mathrm{~d} \theta^{2}\right)}{r^{2}\left(1+\left(\frac{r_{0}}{r}\right)^{2 \kappa}\right)}$

in which $r_{0}$ is a constant parameter. The solution given in [13] for the particular analytic function $\psi(z)=z=r \mathrm{e}^{i \theta}$ (see (4.13) of [13]) can be expressed by 
$\mathrm{d} s^{2}=-\mathrm{d} t^{2}+\frac{\left(\mathrm{d} r^{2}+r^{2} \mathrm{~d} \theta^{2}\right)}{\left(1+\frac{r^{2}}{2 v^{2}}\right)^{2 \chi v^{2}}}$,

in which $\chi$ and $v$ are constant parameters. It can easily be checked that for $\kappa=1,(26)$ coincides, after a time scaling, with (27), upon making the following choice of the parameters:

$\chi=\frac{1}{r_{0}^{2}}=\frac{1}{2 v^{2}}$.

For $\kappa \neq 1$, the two metrics are different. Our metric (26) has also no correspondence with the point particle solution of [13] (see the appendix of [13]). The analogy is valid only for the extended model of particles and confined only to $\kappa=1$.

Our metric (24) represents a regular, non-black hole, static spacetime. The non-zero geometrical quantities are

Ricci scalar: $R=\frac{4 \kappa^{2}}{\cosh ^{2} \rho}$,

Kretschmann scalar: $K=\frac{1}{2} R^{2}$,

and

$R_{\mu \nu} R^{\mu \nu}=K$

with the energy-momentum tensor

$T_{\mu}^{v}=-\frac{R}{2} \delta_{\mu}^{0} \delta_{0}^{\nu}$.

As a result our triplet of scalar fields takes the form

$\phi^{a}(\rho, \theta)=\left(\begin{array}{c}\left(\begin{array}{c}\cos \kappa \theta \\ \sin \kappa \theta\end{array}\right) \frac{2 \sinh \rho}{\cosh \rho} \\ \frac{\sinh ^{2} \rho-1}{\cosh ^{2} \rho}\end{array}\right)$.

This leads, for $\rho=0$, to

$\phi^{a}(0, \theta)=\left(\begin{array}{c}0 \\ 0 \\ -1\end{array}\right)$,

while for $\rho \rightarrow \infty$ we have

$\phi^{a}(\infty, \theta)=\left(\begin{array}{l}0 \\ 0 \\ 1\end{array}\right)$.

It is observed that between $0 \leq \rho<\infty$ the angle $\alpha(\rho)$ shifts from -1 to +1 , which amounts to the case of one kink. It should also be remarked that 'kink' herein is used in the sense of a flip of the $\phi^{3}$ component of the triplet, not in the sense of a light cone tilt. The energy density of the kink is maximum at $\rho=0$, and it decays asymptotically; its energy $E_{\kappa}$ is

$E_{\kappa}=\int_{0}^{\infty} \int_{0}^{2 \pi}\left(-T_{t}^{t}\right) \sqrt{-g} \mathrm{~d} \rho \mathrm{d} \theta=4 \pi|\kappa|$.

\subsection{The harmonic map formulation}

We wish to add, for completeness, that the equation for $\alpha(r)$ can be described as a harmonic map between two Riemannian manifolds $M$ and $M^{\prime}$ [12],

$f^{A}: M \rightarrow M^{\prime}$

which are defined by

$$
\begin{aligned}
M^{\prime}: \mathrm{d} s^{\prime 2} & =\mathrm{d} \alpha^{2}+\sin ^{2} \alpha \mathrm{d} \beta^{2} \\
& =g_{A B}^{\prime} \mathrm{d} f^{A} \mathrm{~d} f^{B} \quad(A, B=1,2)
\end{aligned}
$$

and

$$
\begin{aligned}
M: \mathrm{d} s^{2} & =\frac{\mathrm{d} \rho^{2}}{\kappa^{2}}+\tanh ^{2} \rho \mathrm{d} \theta^{2} \\
& =g_{a b} \mathrm{~d} x^{a} \mathrm{~d} x^{b} \quad(a, b=1,2) .
\end{aligned}
$$

The energy functional of the map is defined by

$E\left(f^{A}\right)=\int g_{A B}^{\prime} \frac{\mathrm{d} f^{A}}{\mathrm{~d} x^{a}} \frac{\mathrm{d} f^{B}}{\mathrm{~d} x^{b}} g^{a b} \sqrt{g} \mathrm{~d}^{2} x$,

which yields, upon variation, the equation for $\alpha(\rho)$. Note that in this map we consider a priori that $\alpha=\alpha(\rho)$ and $\beta=\beta(\theta)$. The degree of the harmonic map $(d)$ is defined in an orthonormal frame $\left\{x^{i}\right\}$ by

$d=\frac{1}{2 \pi} \int \mathrm{d}^{2} x \sin \alpha\left|\frac{\partial(\alpha, \beta)}{\partial\left(x_{1}, x_{2}\right)}\right|=\kappa$,

which equals the topological charge [11].

Although the maps in the original work of Eells and Sampson [12] were considered between unit spheres (in particular $\left.S^{2} \rightarrow S^{2}\right)$ in the present problem our map is from $R^{2} \rightarrow S^{2}$. We must add that the method of harmonic maps was proposed long ago as a model for a non-linear field theory [14]. Einstein's equations of general relativity also follow from a harmonic map formulation [15]. The isometries of the M' metric serves to generate new solutions from known solutions [16]. Unfortunately the non-compact and singular manifolds of general relativity create serious handicaps, which prevented a wider application of the concept of degrees of the maps once they are formulated in terms of harmonic forms.

\section{Conclusion}

In conclusion we comment that the topological properties of field theory were well defined in a flat space background. Due to the singular and non-compact manifolds of general relativity these concepts found no simple applications in a curved spacetime. In this note, similar to the contribution of Ref. [13], we have shown that at least in the 2+1-dimensional spacetime the problem can be overcome. The source of our metric is provided by a triplet of scalar fields, which may find 
applications as multiplets of scalar fields in higher dimensions. It has been shown that the triplet source gives rise to other solutions, such as black holes, besides the topological metric. The interesting feature of such a black hole is that there is an event horizon and, as a result, the Hawking temperature due to the integer $\kappa$ takes discrete multiples of a certain value. As a final remark let us add that it would be interesting to extend our model to $3+1$ dimensions with multi-scalar fields. Technical problems such as the non-linear superposition of sine-Gordon solutions in a curved space, leading to the 'multi-kink' metric, remain to be studied.

Acknowledgments We wish to thank the anonymous referee for directing our attention to Ref. [13].

Open Access This article is distributed under the terms of the Creative Commons Attribution 4.0 International License (http://creativecomm ons.org/licenses/by/4.0/), which permits unrestricted use, distribution, and reproduction in any medium, provided you give appropriate credit to the original author(s) and the source, provide a link to the Creative Commons license, and indicate if changes were made.

Funded by SCOAP . $^{3}$

\section{References}

1. D. Finkelstein, C.W. Misner, Ann. Phys. 6, 230 (1959)

2. J.G. Williams, R.K.P. Zia, J. Phys. A: Math. Nucl. Gen. 6, 1 (1973)

3. J.G. Williams, J. Phys. A: Math. Nucl. Gen. 7, 1871 (1974)

4. D. Finkelstein, G. McCollum, J. Math. Phys. 16, 2250 (1975)

5. M. Vasilic, T. Vukasinac, Class. Quantum Gravity 12, 1995 (1996)

6. J.G. Williams, Gen. Relativ. Gravit. 23, 1995 (1996)

7. T. Klösch, T. Strobl, Phys. Rev. D 57, 1034 (1998)

8. J.G. Williams, Gen. Relativ. Gravit. 30, 27 (1998)

9. M. Bañados, C. Teitelboim, J. Zanelli, Phys. Rev. Lett. 69, 1849 (1992)

10. M. Bañados, M. Henneaux, C. Teitelboim, J. Zanelli, Phys. Rev. D 48, 1506 (1993)

11. J. Honerkamp, A. Patani, M. Schlindwein, Q. Shafi, Lett. Nuovo Cimento 15, 97 (1975)

12. J. Eells, J.H. Sampson, Am. J. Math. 86, 109 (1964)

13. G. Clement, Nucl. Phys. B 114, 437 (1976)

14. C.W. Misner, Phys. Rev. D 18, 4510 (1978)

15. Y. Nutku, Ann. Inst. Henri Poincare XXI, 175 (1974)

16. M. Halilsoy, Phys. Lett. A 84, 404 (1981) 